SCHMIDT, R. A./LEE, T. D.:

Motor Control and Learning. Champaign/ Il.: Human Kinetics 1999, US \$ 59,00.

Das Buch ist in drei Hauptkapitel gegliedert. Im ersten Teil („Introduction to Motor Behavior") werden grundlegende Begriffe und Modelle der Motorikforschung präsentiert. Die Kapitel 2 und 3 beschreiben aktuelle Konzeptionen und empirische Befunde $\mathrm{zu}$ den Bereichen motorische Kontrolle und motorisches Lernen.
StRauss, B. (Hrsg.):

Zuschauer. Göttingen: Hogrefe 1998. 340 S., DM 69,00.

In diesem Buch wird aus vorwiegend sozialpsychologischer Perspektive auf Zuschauer in unterschiedlichen Kontexten (im Sport, aber auch z. B. in Konzert und Theater) eingegangen. Es liefert Grundlagenwissen zur Beurteilung von Zuschauerfluss und Zuschauermotivation. Für Studierende und Lehrer geeignet.

\title{
Carl-Diem-Wettbewerb 1999/2000
}

PD Dr. Kai Röcker und PD Dr. Rainer Wollny heißen die beiden Preisträger des Carl-Diem-Wettbewerbs 1999/2000, den der Deutsche Sportbund alle zwei Jahre ausschreibt. Mit der Verleihung der Carl-Diem-Plakette ist ein Geldpreis in Höhe von jeweils 5000 DM verbunden. 27 Bewerbungen, 14 Habilitationsschriften und 13 Dissertationen wurden eingereicht. Ihre Qualität stellt den Sportwissenschaften ein hervorragendes Zeugnis aus. Es fiel dem Carl-Diem-Kuratorium nicht leicht, über die Rangfolge zu entscheiden.

Die Habilitationsschrift von Kai Röcken (Universität Tübingen) trägt den Titel „Eine neue Betrachtungsweise der zeitlichen Abläufe im Energiestoffwechsel mittels Anwendung eines weiterentwickelten 13C-Dilutionsverfahrens". Rainer Wollny (Universität Heidelberg) reichte seine Habilitationsschrift „Der Einfluss altersbezogener Personenmerkmale auf die Plastizität motorischer Fertigkeitsoptimierungen" ein. Die Fülle hervorragender Arbeiten dokumentiert sich darüber hinaus darin, dass weitere vier Arbeiten auf den Zweiten Platz gesetzt wurden. Diese Platzierung erreichten PD Dr. med. Martin Halle (Göttingen), PD Dr. Hermann Müller (Saarbrücken), PD Dr. Christiane C. Okonek (Bonn) für ihre Habilitationsschriften und Andre Gounot (Strasbourg) für seine Dissertation. Lobende Anerkennungen wurden an PD Dr. Rüdiger Heim (Berlin) und Dr. Jörg Bietz (Marburg) vergeben. Die Preissumme beträgt insgesamt DM $23000,-$, da neben den Ersten Preisen auch die Zweiten Plätze mit jeweils DM 2500,- und die lobenden Anerkennungen mit jeweils 1000 DM ausgestattet sind.

Abgeschlossen wurde der wissenschaftliche Wettbewerb des Deutschen Sportbundes mit der Festakademie am 17. November 2000 an der Humboldt-Universität zu Berlin. Dort wurden den diesjährigen Preisträgern ihre Auszeichnungen von DSB-Präsident Manfred von Richthofen und vom Vorsitzenden des Carl-DiemKuratoriums, Prof. Dr. Dr. h. c. Ommo Grupe, übergeben. Die beiden mit Plaketten ausgezeichneten Arbeiten erhalten einen Druckkostenzuschuss und werden in der Wissenschaftlichen Schriftenreihe des Deutschen Sportbundes veröffentlicht, die im Hofmann-Verlag erscheint. 